\title{
Multisensor Methods to Estimate Thermal Diffusivity
}

\author{
Thomas C. Henderson ${ }^{1}$, Gwen Knight ${ }^{1}$ and Edward Grant ${ }^{2}$
}

\begin{abstract}
Several methods for the estimation of thermal diffusivity are studied in this work. In many application scenarios, the thermal diffusivity is unknown and must be estimated in order to perform other estimation functions (e.g., tracking of the physical phenomenon, or solving other inverse problems like localization or sensor variance, etc.). In particular, we describe:

1) The use of minimization methods (the Golden Mean and Lagarias' simplex) to determine the thermal diffusivity coefficient which when used in a forward heat flow simulation results in the least (vector) distance between the sampled data and the simulated data.

2) The Maximum Likelihood Estimate for thermal diffusivity.

3) The Extended Kalman Filter to recover the thermal diffusivity.
\end{abstract}

We apply these methods to the determination of thermal diffusivity in snow.

\section{INTRODUCTION AND BACKGROUND}

The incorporation of efficient and scalable probabilistic methods into model-based simultaneous state estimation and parameter identification may have a large impact on the exploitation of spatially distributed sensing and computation systems throughout a wide range of scientific domains. Spatially distributed physical phenomena such as temperature, wave propagation, etc., require observation with dynamically located sensors in order to achieve better tuned computational models and simulations. Methods developed here allow for online validation of models through direct sensor observation. Significant problems which must be overcome include the interpolation between measurement data, as well as the estimation of quantities which cannot be directly measured (e.g., thermal diffusivity coefficients). The demonstration of how stochastic partial differential equations can be used to this end should have strong impact on practice in many applications, including the aircraft Structural Health Monitoring (SHM) problem among others.

We describe computational models and sensor networks exploiting appropriate sensing technology for structural health monitoring. We study active monitoring for events requiring damage localization estimation. The structure is excited with heat (or eventually, ultrasound), the signals recorded by distributed sensors, and parameters determined through a reconstruction process.

Our major goal is to provide rigorous Bayesian Computational Sensor Networks to quantify uncertainty in (1) model-

\footnotetext{
*This work was supported by AFOSR-FA9550-12-1-0291

${ }^{1}$ T.C. Henderson and G. Knight are with the School of Computing, University of Utah, SLC, UT, USA; tch at cs.utah.edu, gknight at cs.utah.edu

${ }^{2}$ E. Grant is with the Dept of Electrical and Computer Engineering, North Carolina State University, Raleigh, NC, USA; egrant at ncsu.edu
}

based state estimates incorporating sensor data, (2) model parameters (e.g., diffusion coefficients), (3) sensor node model parameter values (e.g., location, bias), and input source properties (e.g., locations and extent of cracks). This will be achieved in terms of extensions to our recently developed techniques [4], [13], [14]. We call this approach Bayesian Computational Sensor Networks (BCSN). These decentralized methods have reasonable computational complexity and perform Bayesian estimation in general distributed measurement systems (i.e., sensor networks). A model of the dynamic behavior and distribution of the underlying physical phenomenon may be used to obtain a continuous form from the discrete time and space samples provided by a sensor network. Others have recently begun to explore the Bayesian approach for computational simulation; for example, Tinsley [3], [12] who proposes "the systematic treatment of model and data uncertainties and their propagation through a computational model to produce predictions of quantities of interest with quantified uncertainty." The approach is applied to tumor modeling and analysis. Another related work is that of Furukawa [2] who takes location uncertainty into account when localizing defects and "formulates the uncertainties of sensor states stemming from both motion and measurement and allows stochastic identification of defects using recursive Bayesian estimation."

In his detailed analysis of this problem, Sawo considered physical phenomena as either lumped-parameter systems (i.e., depending only on time and described by ODEs) or distributed- parameter systems (i.e., depending on space and time and described by PDEs). His work mainly considered one-dimensional linear PDEs which have the form:

$$
\mathbb{L}\left(\boldsymbol{p}(r, t), \boldsymbol{s}(r, t), \frac{\partial \boldsymbol{p}}{\partial t}, \ldots, \frac{\partial^{i} \boldsymbol{p}}{\partial t^{i}}, \frac{\partial \boldsymbol{p}}{\partial r}, \ldots, \frac{\partial^{i} \boldsymbol{p}}{\partial r^{i}}\right)=0,
$$

where the system state is given by $\boldsymbol{p}(r, t)$ with $r$ the location and $t$ the time. The Bayesian estimation of $\boldsymbol{p}(r, t)$ in terms of (1) requires the conversion of the PDE into a nonlinear finite dimensional model. See Sawo [13] for details.

In several papers, Monde et al. [8], [9], [10], [11] develop analytical methods based on the Laplace transform to obtain a closed form solution for transient temperatures throughout a body using temperature measurements from two locations, estimate thermal diffusivity and conductivity. Woodfield improved these techniques to handle less smooth temperature changes. Closer to our work here, Ukrainczyk [16] minimizes the objective function:

$$
S(a)=[T-u(a)]^{T}[T-u(a)]
$$

where $a$ is the unknown thermal diffusivity, $T$ and $n$ 


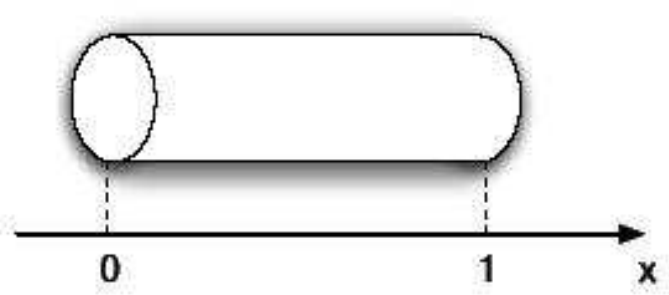

Fig. 1. Heat Flow in a Uniform Rod (adapted from [5]).

are the measured and estimated (based on a guess for a) temperatures, and $a$ is estimated using the LavenbergMarquardt algorithm. Also related to our work is that of Wang et al. [17], [18] who develops a hierarchical Bayesian framework for stochastic inverse heat conduction problems. This includes "(1) stochastic thermal property estimation, (2) automatic selection of the optimal regularization parameter in thermal history reconstruction problems, (3) solution to the IHCP in the presence of uncertainties including errors in thermophysical properties and temperature sensor locations besides temperature measurement noise, and (4) two-scale prior modeling in the estimation of quantities varying in time and space." They use Monte Carlo techniques to estimate the distributions. Our approach differs in that we use the extended Kalman filter. Another closely related work is that of Massard et al. [7] who use a nodal predictive error model with a Bayesian approach.

\section{MINIMIZATION METHODS}

First, we consider a simple approach to the estimation of the thermal diffusivity coefficient. Consider a rod of uniform cross-section and length 1 that is completely isolated except at the ends (see Figure 1). The heat flow is therefore limited to the $x$ direction and the development of the temperature $y$ over time can be described by the following partial differential equation (known as the diffusion equation):

$$
\frac{\partial y}{\partial t}=D \cdot \frac{\partial^{2} y}{\partial x^{2}} \text { with } D=\frac{\kappa}{C \cdot \rho}
$$

where $\kappa$ denotes the thermal conductivity, $C$ the specific heat capacity and $\rho$ the density of the rod.

As mentioned above, Ukrainczyk proposed minimizing the distance between the measurement data samples and the simulation data produced with an estimate of the thermal diffusivity. His method is based on the Levenberg-Marquardt algorithm. We propose the same norm, but two different minimization methods: the Golden Mean method (see [1]) and Matlab's fminsearch (based on Lagarias' simplex method [6]). The first method gets its efficiency from the fact that it uses three points whose endpoints to middle point distances form a golden ratio; this ensures that the interval under consideration is reduced by a constant proportion at each step. The method is then:

initialize 3-point interval

do

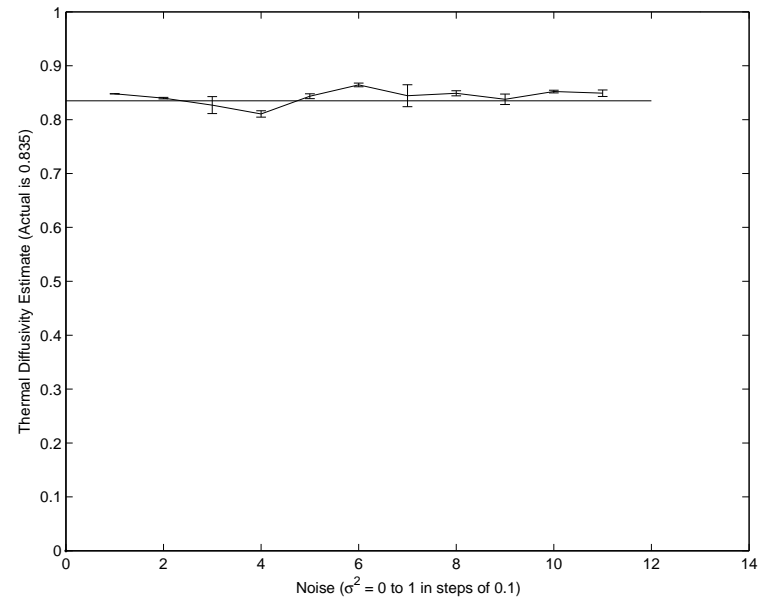

Fig. 2. Golden Mean Thermal Diffusivity Estimate using.

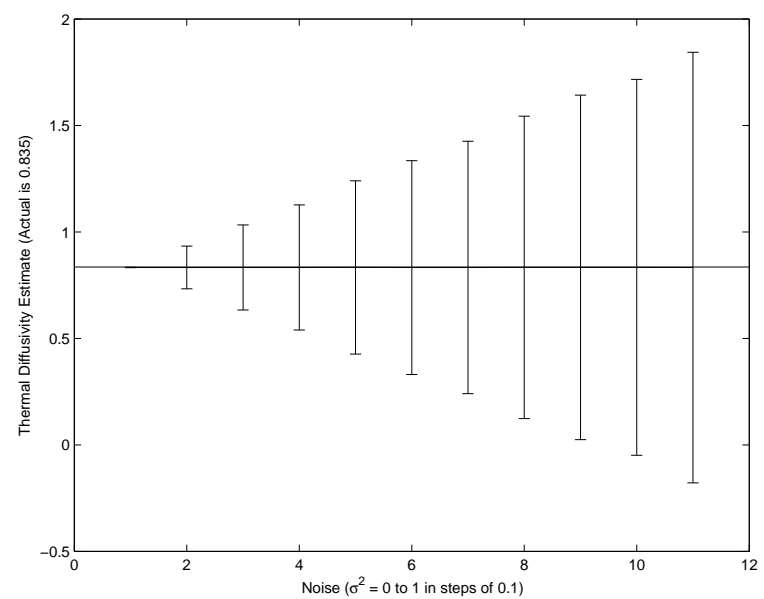

Fig. 3. Thermal Diffusivity Estimate using (a) Golden Mean Method and (b) fminsearch.

get next interval using norm(Z-S)

until (norm (Z-S) <err) or

(interval length<min_len)

Figure 2 shows the robustness of this method over a wide range of noise (variance) in the temperature data; each estimate is the average of 10 simulation runs. Results with the fminsearch method are shown in Figure 3. As can be seen, the Golden Mean method provides less variance, but the estimate is not as good as with fminsearch.

\section{MAXIMUM LIKELIHOOD ESTIMATE}

Given a set of sample temperatures, $S_{i}^{t}$, with time index $t$ and space index $i$, along a rod, with $S_{0}^{t}=T_{0}$, a fixed temperature, and $S_{N+1}^{t}=T_{N+1}$, a fixed temperature, and $S_{i}^{0}=T_{\text {init }}$ for $j=1 \ldots N$. Let $T_{i}^{t}=S_{i}^{t}+\epsilon$ where $\epsilon \sim$ $\mathcal{N}\left(0, \sigma^{2}\right)$ From the heat equation approximation, we have that:

$$
S_{i}^{t}=S_{i}^{t-1}+\lambda\left(S_{i+1}^{t-1}-2 S_{i}^{t-1}+S_{i-1}^{t-1}\right)
$$

for $t=1 \ldots M$ and $i=1 \ldots N$. 
The probability of a specific temperature, $T_{i}^{t}$, given the data and a parameter estimate, $\lambda$ is given by the assumption that a sample is normally distributed about the process model value:

$$
p\left(T_{i}^{t} \mid S, \lambda, \sigma^{2}\right)=\mathcal{N}\left(S_{i}^{t}, \sigma^{2}\right)
$$

Then the likelihood function of the given samples is:

$$
\begin{aligned}
L & =\prod_{t, i=1}^{M, N} \mathcal{N}\left(S_{i}^{t}, \sigma^{2}\right) \\
L & =\prod_{t, i=1}^{M, N} \frac{1}{\sigma \sqrt{2 * \pi}} \exp \left\{-\frac{1}{2 \sigma^{2}}\left(T_{i}^{t}-S_{i}^{t}\right)^{2}\right\}
\end{aligned}
$$

and the log likelihood function is:

$$
\begin{aligned}
& \log L=\sum_{t, i}^{M, N} \log \left(\frac{1}{\sigma \sqrt{2 * \pi}} \exp \left\{-\frac{1}{2 \sigma^{2}}\left(T_{i}^{t}-S_{i}^{t}\right)^{2}\right\}\right) \\
= & \sum_{t, i=1}^{M, N}\left(-\frac{1}{2} \log (2 \pi)-\log (\sigma)-\frac{1}{2 \sigma^{2}}\left(T_{i}^{t}-S_{i}^{t}\right)^{2}\right) \\
= & -\frac{M N}{2} \log (2 \pi)-M N \log (\sigma)-\frac{1}{2 \sigma^{2}} \sum_{t, i=1}^{M, N}\left(T_{i}^{t}-S_{i}^{t}\right)^{2} \\
= & -\frac{M N}{2} \log (2 \pi)-M N \log (\sigma)-\frac{1}{2 \sigma^{2}} \sum_{t, i=1}^{M, N}\left(\left(T_{i}^{t}-S_{i}^{t-1}\right)\right. \\
& \left.-\lambda\left(S_{i+1}^{t-1}-2 S_{i}^{t-1}+S_{i-1}^{t-1}\right)\right)^{2} \\
= & -\frac{M N}{2} \log (2 \pi)-M N \log (\sigma)-\frac{1}{2 \sigma^{2}} \sum_{t, i=1}^{M, N}\left(T_{i}^{t}-S_{i}^{t-1}\right)^{2} \\
& +\frac{\lambda}{\sigma^{2}} \sum_{t, i=1}^{M, N}\left(\left(T_{i}^{t}-S_{i}^{t-1}\right)\left(S_{i+1}^{t-1}-2 S_{i}^{t-1}+S_{i-1}^{t-1}\right)\right) \\
& -\frac{\lambda^{2}}{2 \sigma^{2}} \sum_{t, i=1}^{M, N}\left(S_{i+1}^{t-1}-2 S_{i}^{t-1}+S_{i-1}^{t-1}\right)^{2}
\end{aligned}
$$

Then taking the derivative and setting to zero yields:

$$
\begin{aligned}
& \frac{\partial L}{\partial \lambda}=\frac{1}{\sigma^{2}} \sum_{t, i=1}^{M, N}\left(\left(T_{i}^{t}-S_{i}^{t-1}\right)\left(S_{i+1}^{t-1}-2 S_{i}^{t-1}+S_{i-1}^{t-1}\right)\right) \\
& -\frac{\lambda}{\sigma^{2}} \sum_{t, i=1}^{M, N}\left(S_{i+1}^{t-1}-2 S_{i}^{t-1}+S_{i-1}^{t-1}\right)^{2}
\end{aligned}
$$

Setting equal to zero and solving for $\lambda$ gives:

$$
\lambda=\frac{\sum_{t, i=1}^{M, N}\left(\left(T_{i}^{t}-S_{i}^{t-1}\right)\left(S_{i+1}^{t-1}-2 S_{i}^{t-1}+S_{i-1}^{t-1}\right)\right)}{\sum_{t, i=1}^{M, N}\left(S_{i+1}^{t-1}-2 S_{i}^{t-1}+S_{i-1}^{t-1}\right)^{2}}
$$

Figure 4 shows the ML estimate for a range of noise values.

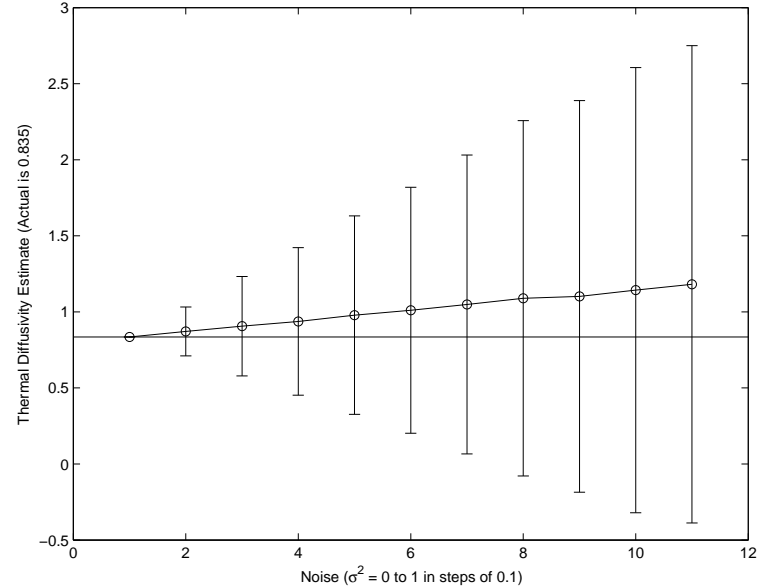

Fig. 4. Thermal Diffusivity Estimate (and variance) using the MLE.

\section{EXTENDED KALMAN FILTER ESTIMATION OF HEAT DIFFUSIVITY}

The Extended Kalman Filter is widely used in estimation; see [15] for an introduction. We use it here to solve for the thermal diffusivity by adding it to the state variable. The heat equation gives rise to the following relation $\left(x_{i}^{t}\right.$ is the temperature at location $i$ at time $t$ ):

$$
x_{i}^{t}=x_{i}^{t-1}+\lambda\left(x_{i+1}^{t-1}-2 x_{i}^{t-1}+x_{i-1}^{t-1}\right)+\epsilon
$$

at locations where the temperature changes, and:

$$
x_{i}^{t}=x_{i}^{t-1}+\epsilon
$$

where the temperature is constant. $\epsilon \sim \mathcal{N}\left(0, \sigma_{p}^{2}\right)$ distribution. Thus:

$$
\bar{x}^{t}=A \bar{x}^{t-1}+\bar{\epsilon}
$$

and

$$
A=\left[\begin{array}{cccccc}
1 & & & & & \\
\lambda & 1-2 \lambda & \lambda & & \\
& & \ddots & & & \\
& & & \lambda & 1-2 \lambda & \lambda \\
& & & & & 1
\end{array}\right]
$$

Temperature can then be tracked with a standard Kalman filter since this is a linear relation. However, an Extended Kalman Filter is needed once we estimate the thermal diffusivity parameter, $\lambda$. This requires adding a state parameter, $x_{N+2}^{t}$, for $\lambda$ and providing the nonlinear update functions. This gives us the two prediction equations:

$$
\begin{aligned}
& g_{i}\left(x_{i}^{t-1}\right)=x_{i}^{t-1}+\frac{\delta t\left(x_{i+1}^{t-1}-2 x_{i}^{t-1}+x_{i-1}^{t-1}\right) x_{n}^{t-1}}{\delta x^{2}} \\
& g_{j}\left(x_{j}^{t-1}\right)=x_{j}^{t-1}
\end{aligned}
$$

where $x_{i}^{t}$ is the temperature estimate at time $t$ and location $i$ for $i=1 \ldots N$, and $x_{j}^{t-1}$ is the temperature at the ends of the rod (i.e., $j=0$ and $j=N+1$ ), and the thermal diffusivity estimate (i.e., $j=N+2$ ) at time $t$; this assumes 


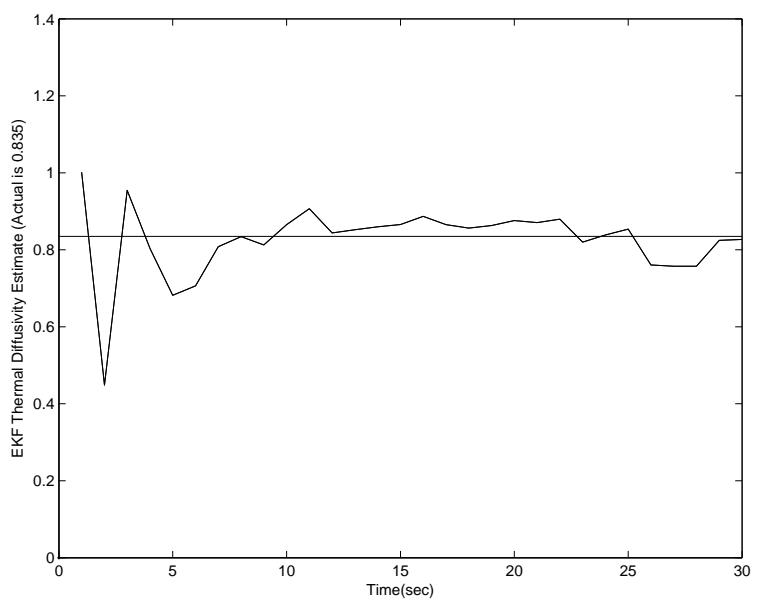

Fig. 5. Tracking Thermal Diffusivity Estimate using the EKF.

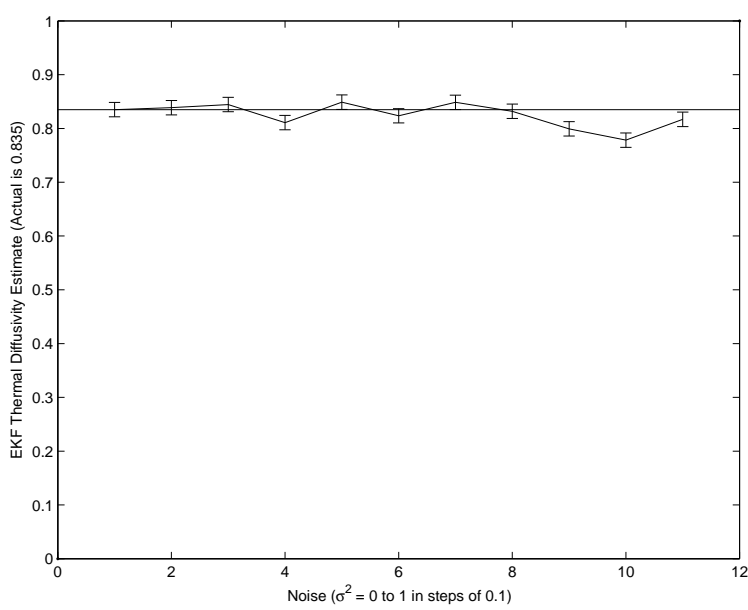

Fig. 6. Thermal Diffusivity Estimate (and Variance) using the EKF.

that there are $N$ locations where the temperature is to be estimated. This leads to the following Jacobian:

$$
\begin{aligned}
& \frac{\partial g_{i}}{\partial x_{i}}=1-2 x_{N+2} \delta t / \delta x^{2} \\
& \frac{\partial g_{i}}{\partial x_{N+2}}=\delta t\left(x_{i+1}-2 x_{i}+x_{i-1}\right) / \delta x^{2}
\end{aligned}
$$

Figure 5 shows the convergence of the estimate for the thermal diffusivity. Figure 6 shows the result of estimating the thermal diffusivity in the presence of noise.

\section{EXPERIMENTAL 1D DATA}

We have also applied the method to some temperature data that we recorded in the Wasatch Mountains in Utah (the data was taken at the Crest Weather Station at Brighton Ski Resort). There were a total of 23 temperature sensors; see Figure 7 . The values at one end stay pretty constant since this is at the ground level, while the other end varies due to the 24-hour temperature cycle in the air above the snow. We have determined the thermal diffusivity, and Figure 8 shows the fit of a simulated run (based on the thermal diffusivity estimate) versus the measured data.

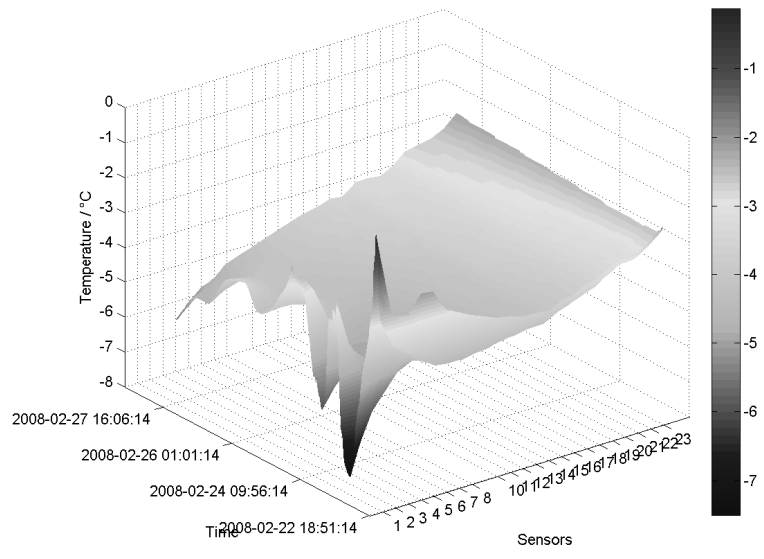

Fig. 7. Data from Temperature Sensors for Multi-Day Period.

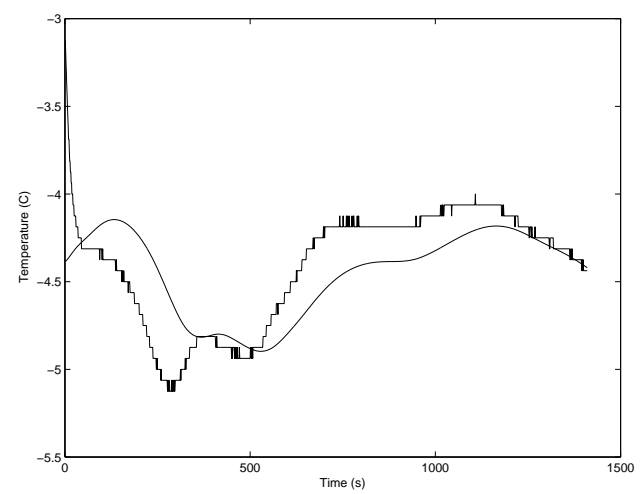

Fig. 8. Overlay of Snow Temperature Data with Simulation (Smooth Curve) using Estimated Thermal Diffusivity.

\section{CONCLUSIONS AND FUTURE WORK}

We are extending the work (1) to handle 2D and other parameters, and (2) to extend the method to use an ultrasound model.

\section{REFERENCES}

[1] S.C. Chapra and R.P. Canale. Numerical Methods for Engineers. McGraw-Hill, Boston, MA, 2010.

[2] T. Furukawa, J. Cheng, S.H. Lim, F. Xu, and R. Shioya. Defect Identification by Sensor Network Under Uncertainties. In Proceedings of the 2010 International Conference on Broadband, Wireless Computing, Communication and Applications, BWCCA '10, pages 155-158, Washington, DC, USA, 2010. IEEE Computer Society.

[3] A. Hawkins-Daarud, S. Prudhomme, K.G. van der Zee, and T. Oden. Bayesian Calibration, Validation, and Uncertainty Quantification of Diffuse Interface Models of Tumor Growth. Technical Report ICES Report 10-44, University of Texas, November 2010.

[4] T.C. Henderson. Computational Sensor Networks. Springer-Verlag, Berlin, Germany, 2009.

[5] T.C. Henderson, C. Sikorski, E. Grant, and K. Luthy. Computational Sensor Networks. In Proceedings of the 2007 IEEE/RSJ International Conference on Intelligent Robots and Systems (IROS 2007), San Diego, USA, 2007.

[6] J.C. Lagarias, J.A. Reeds, M.H. Wright, and P.E. Wright. Convergence Properties of the Nelder-Mean Simplex Method in Low Dimensions. SIAM Jnl of Optimization, 9(1):112-147, 1998.

[7] H. Massard, O. Fudym, H.R.B. Orlande, and J.C. Batsale. Nodal Predictive Error Model and Bayesian Approach for Thermal Diffusivity and Heat Source Mapping. C.R. Mecanique, 338:434-449, 2010. 
[8] M. Monde. Analytical Method in Inverse Heat Transfer Problem using Laplace Transform Technique. Intl Jnl of Heat and Mass Transfer, 43:3965-3975, 2000.

[9] M. Monde, H. Arima, and Y. Mitsutake. Estimation of Surface Temperature and Heat Flux using Inverse Solution for One-dimensional Conduction. Jnl of Heat Transfer, 44:3169-3177, 2003.

[10] M. Monde, M. Kosaka, and Y. Mitsutake. Simple Measurement of Thermal Diffusivity and Thermal Conductivity using Inverse Solution for One-Dimensional Heat conduction. Intl Jnl of Heat and Mass Transfer, 53:5343-5349, 2010.

[11] M. Monde and Y. Mitsutake. A New Estimation Method of Thermal Diffusivity using Analytical Inverse Solution for One-dimensional Heat Transfer Conduction. Intl Jnl of Heat and Mass Transfer, 44:3169-3177, 2001.

[12] T. Oden, R. Mosner, and O. Ghattas. Computer Predictions with Quantified Uncertainty. Technical Report ICES Report 10-39, University of Texas, October 2010.

[13] F. Sawo. Nonlinear State and Parameter Estimation of Spatially Distributed Systems. $\mathrm{PhD}$ thesis, University of Karlsruhe, January 2009.

[14] F. Sawo, T.C. Henderson, C. Sikorski, and U.D. Hanebeck. Sensor Node Localization Methods based on Local Observations of Distributed Natural Phenomena. In Proceedings of the 2008 IEEE International Conference on Multisensor Fusion and Integration for Intelligent Systems (MFI 2008), Seoul, Republic of Korea, August 2008.

[15] S. Thrun, W. Burgard, and D. Fox. Probabilistic Robotics. MIT Press, Cambridge, MA, 2005.

[16] N. Ukrainzydk. Thermal Diffusivity Estimation using Numerical Inverse Solution for 1D Heat Conduction. Intl Jnl of Heat and Mass Transfer, 52:5675-5681, 2009.

[17] J. Wang. Bayesian Computational Techniques for Inverse Problems in Transport Processes. PhD thesis, Cornell University, Cornell, NY, January 2006

[18] J. Wang and N. Zabaras. Using Bayesian Statistics in the Estimation of Heat Source in Radiation. Intl. Jnl of Heat and Mass Transfer, 48:15-29, 2005. 